\title{
Recurrencia de la enfermedad hepática primaria después del trasplante hepático
}

JORGE RAKELA B.

Profesor de Medicina, Mayo Clinic College of Medicine, EE UU de NA.

Texto basado en la conferencia dictada en Sesión pública de la Academia Chilena de Medicina, el 1 de abril de 2009 al ingresar como Miembro Honorario de la Academia.

Recibido el 12 de febrero de 2010. Aceptado el 8 de marzo de 2010 .

Correspondencia a: Jorge Rakela, M.D.

Department of Internal Medicine, Mayo Clinic Arizona, 13400 East Shea Boulevard. Scottsdale, AZ 85259, U.S.A. E-mail: rakela.jorge@mayo.edu

\section{Liver disease recurrence after liver transplantation}

Liver transplantation has become a standard option in the management of patients with end-stage liver disease. It is now evident that the most common etiology of longterm graft dysfunction is the recurrence of the primary liver disease. Autoimmune liver disorders such as autoimmune hepatitis, primary biliary cirrhosis, and primary sclerosing cholangitis recur between 15 to 30\% of the graft recipients. The clinical expression of this recurrence tends to be milder; the diagnosis is only established in many patients by findings in the liver biopsy. This milder clinical expression may be due to the use of immunosuppressive therapy for the prevention of organ rejection and it may also be modulating immune mechanisms that underlie these conditions. The recurrence of hepatitis $C$ virus infection is characterized by an accelerated progression towards cirrhosis and hepatic failure due to the lack of an effective immunoprophylaxis program and an effective antiviral therapy. The recurrence of hepatitis $B$ is uncommon due to the availability on an effective immunoprophylaxis program with effective antiviral agents. The familial amyloidotic polyneuropathy is a genetic condition residing in the hepatocyte that produces a mutation of transthyretin; this abnormal protein is deposited in peripheral nerves, gastrointestinal tract, heart, and kidneys. The liver from these patients, apart from producing this abnormal protein, is otherwise normal, and has been used as an organ for recipients in dire need of a liver transplant, such as patients with hepatocellular carcinoma. This approach is known as "domino" liver transplantation. As these recipients are followed long term, they may develop de novo amyloidosis. In summary, the underlying liver condition that led to end-stage liver disease and liver transplantation may recur after liver transplantation. The clinical expression of the recurrence of the hepatic disease is modulated by the immunosuppression program unless we have an effective immunoprophylaxis and antiviral agents such as in hepatitis $B$.

(Rev Med Chile 2010; 138: 504-510).

Key words: Liver cirrhosis, biliary; Liver transplantation; Recurrence.
E 1 trasplante hepático ha pasado a formar parte de las opciones terapéuticas de rutina en hepatología desde la década de 1980. En una primera etapa del desarrollo del trasplante hepático, la preocupación más importante era el manejo del rechazo agudo y crónico del injerto y de las infecciones en el paciente inmunosuprimido. Con el uso de ciclosporina y luego tacrolimus, estos problemas, aunque aún presentes, se han hecho menos frecuentes y más susceptibles de resolver eficazmente. En 1995, Pappo et $\mathrm{al}^{1}$ de la Universidad de Pittsburgh, demostraron en 51 pacientes que habían recibido un hígado trasplantado y tenían síntomas clínicos y exámenes de laboratorio anormales, que la causa más común de disfunción era la recurrencia de la enfermedad hepática primaria, viral y no-viral. Encontraron que 17 de los 51 los cambios observados eran 
debido a hepatitis viral. Once de los 17 pacientes tenían evidencia de recurrencia de hepatitis viral que había llevado a insuficiencia hepática y había sido la indicación de trasplante. Siete de los 51 pacientes tenían evidencia histológica de recurrencia de la enfermedad original no-viral. Hepatitis B y C, enfermedad alcohólica, hepatitis autoinmune, cirrosis biliar primaria y colangitis esclerosante primaria recidivaron en el hígado injertado. Estos autores demostraron que en 35\% de los injertos con evidencia de disfunción, ésta es debida a la recidiva de la enfermedad primaria. En la etapa actual del desarrollo del trasplante hepático, la causa más común de disfunción tardía del hígado trasplantado es la recidiva de la enfermedad hepática primaria.

La expresión clínica y el manejo terapéutico de la recidiva de la enfermedad primaria dependerá de si esta enfermedad primaria es una enfermedad autoinmune, viral, o genética. Las tres enfermedades autoinmunes más comunes -hepatitis autoinmune, cirrosis biliar primaria y colangitis esclerosante primaria- son susceptibles de ser tratadas con trasplante hepático en sus respectivas fases terminales. La supervivencia de pacientes e injertos es excelente; sin embargo, a medida que estos pacientes trasplantados son observados por tiempos prolongados, se ha hecho evidente que la condición primaria puede recidivar. La recurrencia de la enfermedad autoinmune ocurre en 15 a 25\%, con un intervalo medio de 2 a 5 años.

\section{Hepatitis autoinmune}

Los pacientes con hepatitis autoinmune (HAI) que requieren de trasplante hepático tienen excelentes resultados en términos de supervivencia a 5 años del injerto y del paciente.

La experiencia de la Clínica Mayo, con 41 enfermos con HAI tipo 1 en fase terminal, se publicó en el año $2001^{2}$. La recidiva de la HAI fue observada en 7 de los 41 (17\%) pacientes trasplantados y se hizo evidente clínicamente en un tiempo promedio de 4,6 años después del trasplante hepático. La gran mayoría de estos enfermos fueron asintomáticos y el diagnóstico se estableció debido a elevación de aminotransferasas y una biopsia hepática con características típicas de HAI -infiltrado de linfocitos y plasmocitos, y compromiso lobulillar- presencia de anticuerpos antinucleares y anti-fibra muscular lisa, elevación de gammaglobulina y respuesta terapéutica a esteroides. La supervivencia a 5 años fue de $86 \%$, que no fue distinta de la supervivencia de pacientes que no demostraron recidiva de la HAI. Esta recidiva era más frecuente en asociación con los antígenos de histocompatibilidad HLADR3 y HLA-DR4. Las manifestaciones clínicas favorables y el excelente pronóstico en términos de supervivencia de pacientes y del injerto puede deberse a una detección precoz de la enfermedad, gracias al uso rutinario de la biopsia hepática y el uso de la terapia inmunosupresora que no sólo evitaría el rechazo agudo o crónico sino que también ayudaría a mitigar la expresión clínica de la recurrencia de la enfermedad. Tacrolimus, ciclosporina y mofetil micofenolato se han utilizado en pacientes inmunocompetentes que son refractarios a esteroides, con excelentes resultados ${ }^{3}$.

Una serie más reciente de Vogel et al en $2004^{4}$ demostró una recurrencia de $32 \%$ y una supervivencia a 5 años de $78,2 \%$ que no fue distinta de la de aquellos sin recidiva de HAI. Estos autores observaron una mayor frecuencia de rechazo agudo en estos enfermos cuando los compararon con pacientes con etiologías genéticas.

\section{Cirrosis biliar primaria $(\mathrm{CBP})$}

Una revisión reciente de publicaciones que reportaron sobre la recidiva de la cirrosis biliar primaria (CBP) después del trasplante hepático, acumuló un total de 1.241 pacientes $^{5}$. La incidencia de la recidiva de CBP fue de $16 \%$. La mayoría de los pacientes fueron mujeres (90\%). El seguimiento después del trasplante hepático fue de 69 meses y el tiempo medio hasta la recidiva fue de 46,5 meses. Los criterios histológicos para establecer la presencia de recidiva de CBP tienen que ser muy rigurosos puesto que los hallazgos histológicos de rechazo agudo, y en especial crónico, y de la enfermedad de injerto contra huésped pueden ser confundidos con la recidiva de la CBP. Los títulos de anticuerpos antimitocondriales disminuyen durante el primer año después del trasplante para luego subir a los niveles previos al trasplante, e inclusive exceder estos niveles a pesar de la terapia inmunosupresora. Este fenómeno indicaría indirectamente que el proceso patológico responsable por el desarrollo de la enfermedad sigue presente.

Diversos grupos han observado tendencia a 
una mayor incidencia de recidiva de CBP con el uso de tacrolimus como agente inmunosupresor de base ${ }^{5}$. Estas observaciones ha llevado a varios programas de trasplante hepático a preferir el uso de ciclosporina como agente inmunosupresor de base en estos pacientes. También se ha sugerido que una mayor edad del donante y receptor, tiempo de isquemia fría o tibia prolongado facilitarían la recurrencia de la CBP; estas observaciones son más controversiales ${ }^{6}$. Ha sido de gran interés, al igual que con HAI, la observación que la recidiva de $\mathrm{CBP}$ es de pronóstico excelente en términos de supervivencia del paciente y del injerto. Es un evento muy poco frecuente que estos pacientes con recidiva de la enfermedad autoinmune requieran de un nuevo trasplante.

Una vez que el diagnóstico de CBP recurrente se ha establecido, es aconsejable administrar a la paciente ácido ursodeoxicólico. El grupo de la Clínica Mayo observó que en un período de 36 meses, 52\% de los 38 pacientes tratados con ácido ursodeoxicólico normalizaron sus niveles de fosfatasas alcalinas y alanina aminotransferasa séricas, en contraste con que sólo $22 \%$ de los pacientes no tratados hubo esa normalización ${ }^{7}$. Sin embargo, no hubo una diferencia significativa en la progresión del daño histológico en ambos grupos. El grupo mencionado no observó una influencia evidente de este tratamiento en la supervivencia de los pacientes e injertos durante el período observado. No hay evidencia para justificar el uso de esteroides para prevenir o tratar la recurrencia de CBP. Actualmente hay un estudio multicéntrico, controlado, para evaluar el uso profiláctico del ácido ursodeoxicólico luego del trasplante en un afán de prevenir la recurrencia de esta enfermedad.

\section{Colangitis esclerosante primaria (CEP)}

La CEP es una enfermedad crónica hepática de probable etiología inmune; aunque el defecto inicial aún no se ha definido. Alrededor de 5\% de los pacientes que reciben trasplante hepático en Estados Unidos de Norteamérica es por esta enfermedad. La supervivencia de los pacientes trasplantados a 5 años es alrededor de $75 \%$. Es así que se ha hecho evidente que esta condición recidiva clínicamente e histológicamente en el hígado trasplantado.

Los criterios clínicos y patológicos de recu- rrencia de CEP fueron definidos por Graziadei et $\mathrm{al}^{8}$. Estos autores estudiaron 150 pacientes con el diagnóstico de CEP y que fueron trasplantados. El seguimiento después de trasplante fue de 55 meses. Definieron criterios radiológicos e histológicos estrictos; le dieron especial atención a la presencia de estenosis biliares no-anastomóticas intrahepáticas y/o extrahepáticas con dilataciones cortas, saculares, que aparecen $>90$ días después del trasplante. La presencia de colangitis fibrosa $o$ lesiones fibro-obliterativas de conductillos biliares grandes. Además se debe excluir la presencia de trombosis de la arteria hepática, rechazo crónico con ductopenia, incompatibilidad $\mathrm{ABO}$ de donante-receptor, presencia de estenosis en la línea de anastomosis biliar y el desarrollo de estenosis no-anastomótica antes de 90 días. Con la utilización de estos criterios estrictos, encontraron que 24 pacientes $(20 \%)$ tenían evidencia de recurrencia de CEP. Estos hallazgos fueron más frecuentes en los pacientes que fueron trasplantados debido a CEP. Los factores que se han visto asociados con la recurrencia de CEP incluyen una incidencia más alta de $\mathrm{HLA}-\mathrm{DRB} 1^{\star} 08$, la edad del receptor, sexo masculino, la presencia de un colon intacto después del trasplante, rechazo agudo recurrente, rechazo agudo refractario a esteroides, y la presencia de un colangiocarcinoma previo al trasplante ${ }^{9-12}$.

La eficacia del uso de ácido ursodeoxicólico o corticosteroides no ha sido demostrada en el manejo de la recurrencia de la CEP.

Sin lugar a dudas que las enfermedades autoinmunes del hígado recidivan después del trasplante; sin embargo, su presentación clínica es mucho más atenuada y una progresión hacia la insuficiencia hepática o descompensación hepática es menos frecuente. Es posible que al ocurrir esta recurrencia en presencia de un programa de inmunosupresión diseñado para prevenir el rechazo del injerto amortiguaría la expresión de fenómenos autoinmunes aberrantes, lo que a su vez llevaría a una expresión clínica más benigna.

Una historia muy distinta es la situación actual con el trasplante hepático en pacientes con cirrosis debida al virus de la hepatitis C (VHC).

\section{Hepatitis viral C}

La recurrencia del VHC es prácticamente universal. El curso clínico hacia el desarrollo de 
cirrosis es acelerado en comparación con los pacientes inmunocompetentes ${ }^{13,14}$. Alrededor de 35\% de los pacientes desarrollan cirrosis en 5-7 años. El $42 \%$ puede presentarse con descompensación de la cirrosis dentro de un año después del diagnóstico de la recidiva de la hepatitis C. La supervivencia del paciente y del injerto en pacientes con hepatitis crónica $\mathrm{C}$ recurrente es inferior cuando se compara con otras causas (VHB, HAI, CBP). Un factor importante en este curso acelerado hacia la cirrosis y descompensación es la progresión acelerada de la fibrosis hepática en estos pacientes. Se estima que en un paciente inmunocompetente infectado con VHC el aumento de fibrosis es 0,1-0,13 unidades de fibrosis por año; en el paciente trasplantado es 0,3-0,6 unidades de fibrosis por año. La expresión clínica e histológica de la recurrencia de la hepatitis C depende de la interacción entre el programa inmunosupresor, la replicación viral y el estadio del sistema inmune del paciente ${ }^{15}$.

La supervivencia a 3 años después de establecerse la insuficiencia hepática es $<10 \%$, comparada con $60 \%$ después de la descompensación en pacientes inmunocompetentes. El tiempo desde la descompensación hasta la muerte del paciente es también más corto en los pacientes trasplantados.

En una publicación reciente de nuestra unidad de trasplante hepático ${ }^{16}$, reportamos que la presencia de fibrosis de estadio $\geq 2$ en la biopsia hepática a los 12 meses, donantes mayores de 45 años, historia de carcinoma hepatocelular y rechazo agudo durante los primeros 6 meses después del trasplante tenían un impacto negativo en la supervivencia del paciente. Otros factores que se han asociado con un curso acelerado de la fibrosis hepática después del trasplante incluyen un tiempo de isquemia prolongado y la infección por citomegalovirus (CMV). Se postuló inicialmente que el uso de donantes vivos pudiera asociarse con un recidiva clínica y patológica más acelerada de la hepatitis C; sin embargo, nuestro grupo demostró que no había diferencia en el curso clínico después de trasplante con donante vivo o con donante cadavérico $^{17}$.

La terapia antiviral con interferón pegilado y ribavirina, que ha sido exitosa en los pacientes inmunocompetentes, ha mostrado resultados inferiores en los pacientes trasplantados. La respuesta virológica sostenida (VHC-RNA negativo en suero 6 meses después de completar el tratamiento) con este programa terapéutico en pacientes sin inmunosupresión es de 50-55\%; en contraste, en pacientes trasplantados esta respuesta es alrededor de $28 \%{ }^{18,19}$. La tendencia actual es tratar la recidiva de la hepatitis $\mathrm{C}$ tan pronto hay evidencia de daño hepático en la biopsia hepática, con fibrosis $\geq 2$ de 4. Se ha propuesto tratar a estos pacientes en forma profiláctica tan pronto son trasplantados y hay evidencia virológica de re-infección con el virus de la hepatitis C; este enfoque terapéutico se está evaluando en un estudio controlado, multicéntrico.

Un porcentaje menor de pacientes con recidiva de la hepatitis C, 4-6\%, desarrolla una hepatitis colestásica que evoluciona hacia la descompensación hepática dentro de 1 a 6 meses después del injerto ${ }^{15,20}$. Este daño hepático se atribuye a un daño citopático directo del virus sobre el hepatocito ${ }^{15,20,21}$. La eficacia del tratamiento combinado con interferón pegilado y ribavirina es aún menor que en el resto de los enfermos con recurrencia de hepatitis C. Aunque esta complicación es infrecuente, cuando se evalúan los pacientes que necesitan retrasplante por recurrencia de la hepatitis C, el $19 \%$ es debido a una hepatitis colestásica fibrosante de la hepatitis $\mathrm{C}^{22}$. Desafortunadamente, un porcentaje importante de aquellos retrasplantados vuelven a presentar una hepatitis colestásica con un curso rápido a la descompensación hepática.

Con el desarrollo de de la terapia STAT-C (specifically targeted antiviral therapies for hepatitis $C$ ) y el uso de una tercera droga agregada a interferón pegilado y ribavirina existe la posibilidad real de mejorar el porcentaje de pacientes con respuesta viral sostenida y aclaración del HCV-RNA ${ }^{23}$.

\section{Hepatitis B}

Con el uso de un eficaz programa de inmunoprofilaxis con una gammaglobulina específica para el virus B con una droga antiviral oral, la recurrencia de la hepatitis $B$ después del trasplante hepático es menos de 10\%. La supervivencia de estos pacientes es sobre $90 \%$ a los 2 años después del trasplante ${ }^{24-26}$. Los factores que están asociados con la recurrencia de esta infección incluyen un alto nivel de replicación viral caracterizado por la presencia del HBeAg en suero y niveles de HBVDNA sobre 20.000 UI-mL. A su vez si la indicación del trasplante hepático fue una hepatitis fulminante o el paciente tenía un coinfección de virus B y virus delta, la tasa de reinfección es muy baja. Si el 
paciente está infectado con una cepa de virus B resistente a drogas antivirales, es más probable que se reinfecte después del trasplante. Recientemente se ha observado que si el paciente tiene un carcinoma hepatocelular sobrepuesto en una cirrosis, es más probable que tenga una reinfección por virus $\mathrm{B}^{27}$. Faria et al observaron que 11 de 31 pacientes con cirrosis y carcinoma hepatocelular desarrollaron reinfección con virus B, y sólo 3 de 68 de aquellos sin carcinoma hepatocelular.

El programa actual de inmunoprofilaxis es caro y requiere ser administrado de por vida. Recientemente se han publicado resultados preliminares sobre el uso de dos drogas orales, adefovir y lamivudina, sin inmunoglobulina específica para el virus $\mathrm{B}$, con excelentes resultados, comparables al tratamiento establecido ${ }^{28}$.

Otro enfoque ha sido intentar inducir la producción de anticuerpos anti-HBs con la utilización de vacuna contra el virus $\mathrm{B}^{24,29,30}$. El factor limitante de este enfoque ha sido que no todos los pacientes desarrollan títulos alto de anti-HBs y es probable que se necesite una vacuna más antigénica que la que se dispone actualmente ${ }^{30-34}$.

\section{Amiloidosis familiar polineuropática (AFP)}

La AFP es una enfermedad genética que reside en el hepatocito. Es debida a una mutación autosómica dominante del gen de la transtiretina (TTR). La producción de esta proteína anormal ocurre en el hepatocito y se deposita en órganos vitales, nervios periféricos y autonómicos, aparato digestivo, corazón y riñones. Estos hígados, con excepción de la producción de TTR anormal, son funcionalmente normales. La mutación más frecuente es Val30Met. Nuestra experiencia ${ }^{35}$ recientemente publicada consistió de 12 casos de AFP que recibieron un trasplante hepático. Cinco de ellos tenía la mutación más común, Met30 y 7 nonMet30 (Ala60, Tyr70, Gly42, Lys89). Cinco de los pacientes con mutaciones no-Met30 requirieron trasplantes combinados de hígado y corazón en 4 de ellos, y corazón y riñón en uno. La supervivencia después del trasplante fue de 77\%. Se observó mejoría de la neuropatía sensorial en $42 \%$ de los pacientes, del tránsito digestivo en $52 \%$ y de la desnutrición en $40 \%$. Estas mejorías fueron lentas y no completas. Los síntomas cardiovasculares fueron mucho más lentos en mejorar y sólo $21 \%$ de los enfermos. Ésta es la razón para considerar un trasplante combinado de hígado y corazón en estos pacientes.

Hígados de pacientes con AFP han sido utilizados como trasplante "dominó" en candidatos a trasplante hepático con carcinoma hepatocelular que tienen la perspectiva de esperar por un trasplante un tiempo que los pone en riesgo de progresión de la enfermedad, que puede impedir realizar un trasplante.

En estos receptores de hígados "dominó" proveniente de donantes con AFP, se ha observado la acumulación de fibras de amiloide anormales en mucosa gástrica ${ }^{36}$ y con el tiempo también evidencia de compromiso de la conducción nerviosa por acumulación de fibras de amiloide en los nervios periféricos. Yamamoto et $\mathrm{al}^{37}$ recientemente comunicaron que en 20 pacientes que fueron receptores de un hígado "dominó" proveniente de un donante con AFP, 3 de ellos desarrollaron evidencia de compromiso de la conducción nerviosa determinada por electroneurografía. Estas alteraciones se evidenciaron 2 a 5 años después del trasplante. Estas observaciones sugieren que la amiloidosis de novo puede ocurrir antes de los 15 años que se pensaba si estos pacientes siguen la historia natural de la enfermedad.

Defectos genéticos como el causante de AFP son resueltos completamente por el trasplante hepático. Esperamos que estos defectos puedan ser corregidos en el futuro a través de terapia génica y no requerir de un tratamiento tan drástico como el trasplante hepático.

En resumen, a medida de que los pacientes que tienen trasplante hepático viven más años por las mejorías evidentes en técnicas quirúrgicas, manejo de tratamiento intensivo, programas de inmunosupresión y tratamiento de infecciones, una nueva patología va emergiendo. La enfermedad primaria, que llegó a su fase terminal, recurre. La expresión clínica de esta recurrencia se ve modificada por el programa de inmunosupresión. En el caso de enfermedades autoinmunes, esta expresión clínica tiende a ser más benigna que la enfermedad original. En el caso de enfermedades virales como VHC, el cuadro clínico, debido al programa de inmunosupresión, progresa en forma acelerada porque no disponemos de programas de inmunoprofilaxis eficaces.

Esperamos que en los años venideros podremos disponer de medicamentos eficaces para pre- 
venir la re-infección por VHC como disponemos actualmente para prevenir la re-infección con virus de la hepatitis B.

Defectos genéticos como el de la AFP que reside en el hepatocito son completamente corregidos por el trasplante hepático, con el inconveniente que el paciente tiene que llevar un programa de inmunosupresión de por vida. La solución de estos cuadros clínicos residirá con seguridad en terapias génicas eficaces y sin efectos colaterales serios.

El trasplante hepático provee una situación única para investigar los mecanismos que llevan a enfermedad hepática, puesto que a través de este procedimiento extirpamos el órgano "diana" pero en la gran mayoría de los casos no modificamos fundamentalmente los mecanismos patológicos que llevan a la enfermedad en primer lugar.

\section{Referencias}

1. Pappo O, Ramos H, Starzl TE, Fung JJ, Demetris AJ. Structural integrity and identification of causes of liver allograft dysfunction occurring more than 5 years after transplantation. Am J Surg Pathol 1995; 19: 192-206.

2. González-Koch A, Czaja AJ, Carpenter HA, Roberts SK, Charlton MR, Porayko MK, et al. Recurrent autoimmune hepatitis after orthotopic liver transplantation. Liver Transpl 2001; 7: 302-10.

3. Larsen FS, Vainer B, Eefsen M, Bjerring PN, Adel Hansen B. Low-dose tacrolimus ameliorates liver inflammation and fibrosis in steroid refractory autoimmune hepatitis. World J Gastroenterol 2007; 13: 3232-6.

4. Vogel A, Heinrich E, Bahr MJ, Rifai K, Flemming P, Melter M, et al. Long-term outcome of liver transplantation for autoimmune hepatitis. Clin Transplant 2004; 18: 62-9.

5. Manjushree G, Rekha C, Vijayan B. Recurrence of autoimmune liver disease after liver transplantation: A systematic review. Liver Transplantation 2006; 12: 1813-24.

6. Guy JE, Qian P, Lowell JA, Peters MG. Recurrent primary biliary cirrhosis: peritransplant factors and ursodeoxycholic acid treatment post-liver transplant. Liver Transpl 2005; 11: 1252-7.

7. Charatcharoenwitthaya P, Pimentel S, Talwalkar JA, Enders FT, Lindor KD, Krom RA, et al. Long-term survival and impact of ursodeoxycholic acid treatment for recurrent primary biliary cirrhosis after liver transplantation. Liver Transpl 2007; 13: 1236-45.

8. Graziadei IW, Wiesner RH, Batts KP, Marotta PJ, Larusso NF, Porayko MK, et al. Recurrence of primary sclerosing cholangitis following liver transplantation. Hepatology 1999; 29: 1050-6.

9. Cholongitas E, Shusang V, Papatheodoridis GV, Marelli L, Manousou P, Rolando N, et al. Risk factors for recurrence of primary sclerosing cholangitis after liver transplantation. Liver Transpl 2008; 14: 138-43.

10. Alexander J, Lord JD, Yeh MM, Cuevas C, Bakthavatsalam R, Kowdley KV. Risk factors for recurrence of primary sclerosing cholangitis after liver transplantation. Liver Transpl 2008; 14: 245-51.

11. Alabraba E, Nightingale P, Gunson B, Hubscher S, Olliff $S$, Mirza D, et al. A re-evaluation of the risk factors for the recurrence of primary sclerosing cholangitis in liver allografts. Liver Transpl 2009; 15: 330-40.

12. Campsen J, Zimmerman MA, Trotter JF, Wachs M, Bak $\mathrm{T}$, Steinberg T, et al. Clinically recurrent primary sclerosing cholangitis following liver transplantation: a time course. Liver Transpl 2008; 14: 181-5.

13. Araya V, Rakela J, Wright T. Hepatitis C after orthotopic liver transplantation. Gastroenterology 1997; 112: 57582.

14. Gane E. The natural history and outcome of liver transplantation in hepatitis $C$ virus-infected recipients. Liver Transpl 2003; 9: S28-34.

15. Demetris AJ. Evolution of hepatitis $\mathrm{C}$ virus in liver allografts. Liver Transpl 2009; 15 (Suppl 2): S35-41.

16. Gallegos-Orozco JF, Amir Y, Brie N, Bashar AA, Thomas JB, Elizabeth JC, et al. Natural history of post-liver transplantation hepatitis C: A review of factors that may influence its course. Liver Transplant 2009; 15: 1872-81.

17. Guo L, Orrego M, Rodríguez-Luna H, Balan V, Byrne T, Chopra K, et al. Living donor liver transplantation for hepatitis C-related cirrhosis: no difference in histological recurrence when compared to deceased donor liver transplantation recipients. Liver Transpl 2006; 12: 5605.

18. Rodríguez-Luna H, Khatib A, Sharma P, De Petris G, Williams JW, Ortiz J, et al. Treatment of recurrent hepatitis $\mathrm{C}$ infection after liver transplantation with combination of pegylated interferon alpha $2 \mathrm{~b}$ and ribavirin: an open-label series. Transplantation 2004; 77: 190-4.

19. Carrion JA, Navasa M, García-Retortillo M, García-Pagan JC, Crespo G, Bruguera M, et al. Efficacy of antiviral therapy on hepatitis $\mathrm{C}$ recurrence after liver transplantation: a randomized controlled study. Gastroenterology 2007; 132: 1746-56.

20. Doughty AL, Spencer JD, Cossart YE, Mccaughan GW. Cholestatic hepatitis after liver transplantation is associated with persistently high serum hepatitis $C$ virus RNA levels. Liver Transpl Surg 1998; 4: 15-21.

21. Demetris AJ, Eghtesad B, Marcos A, Ruppert K, Nalesnik 
MA, Randhawa P, et al. Recurrent hepatitis $\mathrm{C}$ in liver allografts: prospective assessment of diagnostic accuracy, identification of pitfalls, and observations about pathogenesis. Am J Surg Pathol 2004; 28: 658-69.

22. Mccashland T, Watt K, Lyden E, Adams L, Charlton M, Smith AD, et al. Retransplantation for hepatitis C: results of a U.S. multicenter retransplant study. Liver Transpl 2007; 13 (9): 1246-53.

23. Pawlotsky JM, Chevaliez S, Mchutchison JG. The hepatitis $\mathrm{C}$ virus life cycle as a target for new antiviral therapies. Gastroenterology 2007; 132: 1979-98.

24. Coffin CS, Terrault NA. Management of hepatitis B in liver transplant recipients. J Viral Hepat 2007; 14 (Suppl 1): 37-44.

25. Papatheodoridis GV, Cholongitas E, Archimandritis AJ, Burroughs AK. Current management of hepatitis B virus infection before and after liver transplantation. Liver Int 2009; 29: 1294-305.

26. Terrault N, Roche B, Samuel D. Management of the hepatitis B virus in the liver transplantation setting: a European and an American perspective. Liver Transpl 2005; 11: 716-32.

27. Faria LC, Gigou M, Roque-Afonso AM, Sebagh M, Roche B, Fallot G, et al. Hepatocellular carcinoma is associated with an increased risk of hepatitis B virus recurrence after liver transplantation. Gastroenterology 2008; 134: 1890-9; quiz 2155.

28. Angus PW, Patterson SJ, Strasser SI, Mccaughan GW, Gane E. A randomized study of adefovir dipivoxil in place of HBIG in combination with lamivudine as postliver transplantation hepatitis B prophylaxis. Hepatology 2008; 48: 1460-6.

29. Rimola A, Sánchez-Fueyo A. Hepatitis B vaccination after liver transplantation as a strategy to discontinue prophylaxis with hepatitis B immune globulin. J Hepatol 2004; 40: 722-4.

30. Sánchez-Fueyo A, Martínez-Bauer E, Rimola A. Hepatitis $B$ vaccination after liver transplantation. Hepatology 2002; 36: 257-8; author reply 258-9.

31. Rosenau J, Hooman N, Hadem J, Rifai K, Bahr MJ, Philipp G, et al. Failure of hepatitis B vaccination with conventional HBsAg vaccine in patients with continuous HBIG prophylaxis after liver transplantation. Liver Transpl 2007; 13: 367-73.

32. Gunther M, Neuhaus R, Bauer T, Jilg W, Holtz JA, Bienzle U. Immunization with an adjuvant hepatitis B vaccine in liver transplant recipients: antibody decline and booster vaccination with conventional vaccine. Liver Transpl 2006; 12: 316-9.

33. Poland GA, Poterucha JJ. Hepatitis B immunization after liver transplantation: what is the answer? Liver Transpl 2005; 11: 1181-3.

34. Angelico M, Di Paolo D, Trinito MO, Petrolati A, Araco A, Zazza S, et al. Failure of a reinforced triple course of hepatitis B vaccination in patients transplanted for HBV-related cirrhosis. Hepatology 2002; 35: 176-81.

35. Sharma P, Perri RE, Sirven JE, Zeldenrust SR, Brandhagen DJ, Rosen CB, et al. Outcome of liver transplantation for familial amyloidotic polyneuropathy. Liver Transpl 2003; 9: 1273-80.

36. Takei Y, Gono T, Yazaki M, Ikeda S, Ikegami T, Hashikura $\mathrm{Y}$, et al. Transthyretin-derived amyloid deposition on the gastric mucosa in domino recipients of familial amyloid polyneuropathy liver. Liver Transpl 2007; 13: 215-8.

37. Yamamoto S, Wilczek HE, Iwata T, Larsson M, Gjertsen $\mathrm{H}$, Soderdahl G, et al. Long-term consequences of domino liver transplantation using familial amyloidotic polyneuropathy grafts. Transpl Int 2007; 20: 926-33. 